\title{
Bayesian modeling of pharmaceutical data addressing the average effect of bivariate parameters of interest in a bioequivalence framework
}

\author{
$\underline{\text { Al Bartolucci }}{ }^{1}$, Sejong Bae $^{2}$ and Karan P. Singh ${ }^{2}$ \\ ${ }^{1}$ Department of Biostatistics, School of Public Health, University of Alabama at \\ Birmingham, Birmingham, Alabama 35294-0022, USA \\ ${ }^{2}$ Department of Biostatistics, School of Public Health, University of North Texas Health Science Center, Fort \\ Worth, Texas 76107-2699, USA
}

\begin{abstract}
Bioavailability is the rate and extent to which the active ingredient or active moiety is absorbed from a drug product and becomes available at the site of action. For drug products that are not intended to be absorbed into the bloodstream, bioavailability may be assessed by measurements intended to reflect the rate and extent to which the active ingredient or active moiety becomes available at the site of action. Bioequivalence according to regulatory requirements is the absence of a significant difference in the rate and extent to which the active ingredient or active moiety in pharmaceutical equivalents or pharmaceutical alternatives becomes available at the site of drug action when administered at the same molar dose under similar conditions in an appropriately designed study. The study is basically a crossover comparison of absorption of two compounds to determine the maximum absorption (Cmax) and the area under the curve (AUC) of the two compounds and determining if their mean difference falls into a specified confidence region of bioequivalence.
\end{abstract}

Typically, generic drug formulations are often prescribed for 'brand name' (i.e.standard) formulations and given by pharmacists in an effort to reduce the cost of prescription-drug therapy. In most regions, generic replacement is allowed and encouraged, provided that the generic formulation is deemed to be therapeutically equivalent to the standard formulation by the United States Food and Drug Administration (FDA). The FDA publishes a list of drug products and equivalents, which is entitled, Approved Drug Products with Therapeutic Equivalence Evaluations. This is commonly known as the Orange Book. The FDA's designation of 'therapeutic equivalence' indicates that the generic formulation is (among other things) bioequivalent to the standard formulation and usually indicates that the FDA expects that the formulations are likely to have equivalent clinical effect and, in addition, have no difference in their potential for adverse effects. The 1984 Amendments to the Drug Price Competition and Patent Term Restoration Act require that manufacturers seeking approval of generic formulations submit to the FDA data demonstrating bioequivalence to the reference or standard drug product

One of the primary considerations for bioequivalence is the drug's amount and rate of drug absorption and eventually expulsion. The approach taken in this paper is to model this system of absorption via a Bayesian technique taking into account the overall mean treatment and crossover period effects as well as the sequence in which the compounds are given to an individual in a properly designed trial. For each of the parameters of interest we will determine if those values across the two compounds satisfy the boundaries of an acceptable posterior credible region. Typically the Cmax and the AUC are treated independently in the analysis. For most applications to date this has been considered the standard strategy. As an added consideration, we will examine the consequences of a possible correlation between the Cmax and the AUC. In a typical crossover design, subjects are randomly separated into two groups of equal numbers. In the time period 1 the reference formulation is given to group A and the test to group B. During the 'washout period' it is assumed that there is sufficient time duration to allow elimination of the drug administered in the first period. Then in the time period 2 the reference formulation is given to group B and the test to group A. The crossover design allows one to statistically account for "period or sequence effects". We will examine all the variables of interest using prior input empirical results from previous work. The model being investigated is a multivariate model with prior structural considerations on the correlation parameter in an attempt to properly account for the posterior correlation of the parameters of interest, usually the posterior mean of the AUC and the Cmax. Advantages and limitations of our approach will be discussed.

Key Words: Bioequivalence, Correlation structures, Prior to posterior, Sequence, Period, Treatment effects, Bayesian 
Al Bartolucci et al., Bayesian modeling of pharmaceutical data addressing the average effect of bivariate...

\section{INTRODUCTION}

The issue of bioequivalence testing continues to be of great interest to the pharmaceutical and biotechnology industry. This is especially evident since the marketed products having the same amounts of the drug may exhibit marked differences in their therapeutic responses (Westlake, 1981). Ghosh and Rosner (2007) give an excellent summary of the history of bioequivalence which we outline here. These authors as well as others have noted that on the basis of simple pharmacokinetic concepts and parameters, bioavailability and bioequivalence studies have been established as acceptable surrogates for expensive, complicated and lengthy clinical trials. These trials are used worldwide to establish and confirm consistent product quality, as well as reliable and therapeutically effective performance of marketed dosage formulation. It has been established by Chow and Liu (2000) that three situations have arisen in which bioequivalence studies are required. They are: (i) when the proposed marketed dosage form is different from that used in pivotal clinical trials, (ii) when significant changes are made in the manufacture of the marketed formulation, and (iii) when a new generic formulation is tested against the standard or reference marketed product. A bioequivalence study is an experiment to compare a test product (B) to a reference product (A). Bioequivalence studies compare both the rate and extent of absorption of various drug formulations with the standard (reference) product on the basis that if two formulations exhibit similar drug concentration-time profiles in the blood/plasma, they should exhibit similar therapeutic effects. For a new and not yet approved generic dosage formulation to be marketed and accepted as therapeutically effective in relation to the reference product, it must have established bioequivalence with the innovator product, in vivo. The determination of bioequivalence is, thus, very important in the pharmaceutical industry because regulatory agencies like the U.S. Food and Drug Administration (FDA) allow a generic drug to be marketed only if its manufacturer can demonstrate that the generic product is bioequivalent to the reference product. According to FDA regulations (2003), a valid statistical evaluation of a bioequivalence trial is essential in order to guarantee the safety and efficacy of the generic drug products. Bioequivalence studies usually proceed by administering dosages to subjects and measuring the concentration of the drug in the blood just before and at set times after its administration.

These concentration-by-time measurements result geometrically in a polygonal curve and measurements of the drug's pharmacokinetics, like AUC (area under curve), Cmax (maximum concentration) and Tmax (time to maximum concentration) are calculated. For statistical analysis, and as comparative purposes, these measures are taken as the response variables. Regulatory guidelines have also suggested the consideration of average bioequivalence (ABE) (Berger et. al., 1996). As pointed out in Ghosh and Rosner (2007), ABE requires equivalence between the population means of the pharmacokinetic measurements for the reference and test formulations. Recently,, the FDA (2003) supplemented ABE with two more criteria described as , population bioequivalence (PBE) and individual bioequivalence (IBE). These new criteria have also been the subject of dispute. Also the PBE is sometimes confused as the probability of bioequivalence. This has more relevance in the Bayesian context which we shall discuss. Nonetheless, the ABE still remains the main criterion for assessing bioequivalence between two formulations. The main advantage of $\mathrm{ABE}$ is its easier interpretation for the intended audiences, including regulators, prescribing physicians, pharmacists, and patients. The criterion of $\mathrm{ABE}$ has also found potential applications in several other areas such as, psychology (Rogers et. al., 1993), chemistry (Roy, 1997), and environmental statistics (Manly, 2004). In this paper, we take a Bayesian approach to assessing ABE as well as other parameters. The key advantage of using a Bayesian approach for bioequivalence trials is the ability of the Bayesian inferential approach to incorporate background or empirical information thought relevant to the clinical question being addressed ( Bartolucci et al. 2008). Breslow (1990) presented arguments that bioequivalence is a perfectly natural concept to be subjected to Bayesian analysis. Several authors have also advocated a Bayesian approach to average bioequivalence inference (Ghosh and Gonen, 2008). The main idea of all the above methods as we also demonstrate in our work is to find the posterior distribution of the parameter of interest based on non-informative prior distributions for the parameters. A conjugate setting will also work here. All of the existing literature on $\mathrm{ABE}$, however, relies heavily on a normality assumption. The normality assumption in a bioequivalence trial may not always be true, however, and the inference can be misleading. Bolton (1991) discussed that the normality assumption in a bioequivalence trial may lack robustness against outliers and skewness. Usually a bioequivalence trial is conducted with a small number of healthy subjects, and it is not always possible to validate the normality assumption. Instead of following a normal distribution, the data from a bioequivalence trial may have a mixture of normal distributions (e.g., diverse populations, such as from pharmacogenetic variation), a distribution with heavier tails, or some other distribution which can not be easily specified. Thus, it became is of practical interest to develop statistical models in $\mathrm{ABE}$ that move beyond the traditional parametric model. This was done rather nicely by Ghosh and Rosner (2007) as well as Ghosh and Gonen (2008). We do not belabor these arguments here.. Our method extends existing methods by allowing for possible heterogeneity of the subjects who are participating in the study by 
Al Bartolucci et al., Bayesian modeling of pharmaceutical data addressing the average effect of bivariate...

allowing for a random subjects effect. Our next focus is to apply the approach of Ghosh and Gonen (2008) to investigate the effect of the correlation structure on the AUC and Cmax. It is often assumed that these two parameters are independent. Thus an ABE is attained for each separately under the assumption of independence. We investigate various prior structures on the correlation from the beta family of distributions and determine first of all the strength of the correlation and then the effect of the correlation on the outcome using a bivariate model for both the AUC and the Cmax. The limitations of the approach both statistically and from a software perspective will also be presented.

\section{METHODS}

The typical study design employed in bioequivalence studies is the two-treatment, two-period, crossover design In this type of design,subjects are randomly separated into two groups (A and B) of equal number. The reference formulation is administered to group ' $A$ ' in the first study period, and the test formulation is administered to group ' $\mathrm{B}$ ' in the first period. During the second study period, group ' $\mathrm{A}$ ' receives the test formulation and group ' $\mathrm{B}$ ' receives the reference formulation. The first and second study periods are separated by a washout period, which is designed to be of sufficient duration to allow elimination of the drug administered in the first period. Subjects are separated into two groups to allow identification of 'period' or 'sequence' effects in the study results. The sequence is of course A to B or B to A. Without loss of generality we will refer to A as the reference formulation and to B as the test formulation.

As in Ghosh and Gonen (2008) we will consider a $2 \times 2$ crossover design for multivariate ABE endorsed by the FDA (2003). In most bioequivalence trials, a test formulation is compared with the reference formulation in a group of normal, healthy subjects, as recommended by the US FDA (2003). Each participant receives the treatments alternatively in a crossover study. The most commonly used statistical design for comparing average bioequivalence between a test formulation (B) and a reference formulation (A) of a drug is a two-sequence, two-period, crossover design. The following statistical model is usually considered for a $2 \times 2$ crossover design. Consider,

$$
\mathrm{y}_{\mathrm{ijk}}=\mu_{\mathrm{i}, \mathrm{k}}+\mathrm{S}_{\mathrm{i}}+\mathrm{P}_{\mathrm{k}}+\delta_{\mathrm{ij}}+\mathrm{e}_{\mathrm{ijk}}
$$

Here, we consider $y_{\mathrm{ijk}}$ to be the logarithm of response in the ith sequence from the kth period for the jth subject, (i $\left.=1,2 ; \mathrm{j}=1,2, \cdots, \mathrm{n}_{\mathrm{i}} ; \mathrm{k}=1,2\right)$. We use the logarithm, as a standard because often the response measures in a bioequivalence study follow a lognormal distribution, due to skewness. Furthermore, $\mu_{\mathrm{i}, \mathrm{k}}$ is the direct effect of the formulation in the ith sequence that is administered at the kth period, $S_{i}$ is the fixed effect of the ith sequence $\left(S_{1}+\right.$ $\left.\mathrm{S}_{2}=0\right), \mathrm{P}_{\mathrm{k}}$ is the fixed effect of period $\mathrm{k}\left(\mathrm{P}_{1}+\mathrm{P}_{2}=0\right)$. We let $\mathrm{e}_{\mathrm{ijk}}$ be the within subject random error in observing $\mathrm{y}_{\mathrm{ijk} . .}$ The $\delta_{\mathrm{ij}}$ are considered the random subject effect for the ith sequence and kth period. We are assuming they are i.i.d. normal with mean 0 and variance $\sigma^{2}$ and the $e_{i j k}$ are i.i.d. with mean 0 and variance $\sigma_{1}^{2}$ where 1 is the treatment indicator. That is to say, $\mathrm{l}=\mathrm{A}$ if $\mathrm{k}=\mathrm{i}$ and $\mathrm{l}=\mathrm{B}$ otherwise. We assume that in the first period of the first sequence we have the formulation $\mathrm{A}$ then:

$$
\mu_{\mathrm{i}, \mathrm{k}}=\mu_{\mathrm{A}} \text { if } \mathrm{k}=\mathrm{i} \text { and } \mu_{\mathrm{i}, \mathrm{k}}=\mu_{\mathrm{B}} \text { if } \mathrm{k} \neq \mathrm{i} \text {. }
$$

Two compounds or drugs are called average bioequivalent if the population means of the drug-specific AUC's are sufficiently close. We can apply the same to the Cmax. In statistical terms, the problem of ABE is to decide if the difference of two parameters $\Delta=\mu_{\mathrm{A}}-\mu_{\mathrm{B}}$ is close to zero. The usual expression of average bioequivalence is presented as:

$$
\mathrm{H}_{0}: \Delta \leq \theta_{\mathrm{L}} \quad \text { or } \quad \Delta \geq \theta_{\mathrm{U}} \quad \text { vs. Ha }: \theta_{\mathrm{L}}<\Delta<\theta_{\mathrm{U}}
$$

where the lower and upper tolerance limits $\theta_{\mathrm{L}}$ and $\theta_{\mathrm{U}}$ are known constants specified by the sponsor of the trial and the FDA. The limits $\theta_{\mathrm{L}}=\log (0.8)$ and $\theta_{\mathrm{U}}=\log (1.25)$ or $\log (1.2)$ are widely accepted by drug authorities for testing bioequivalence in terms of the parameters of interest. We note that the hypothesis testing set up in (3) is the reverse of the ordinary view of hypothesis testing in the Neyman Pearson context. Whereas a null hypothesis is usually a hypothesis of equivalence, we now consider the lack of equivalence the null hypothesis that we seek to 
Al Bartolucci et al., Bayesian modeling of pharmaceutical data addressing the average effect of bivariate...

disprove. This formulation makes a great deal of sense for bioequivalence trials. Here the type I error is the probability of declaring the drugs to be bioequivalent when in fact they are not. Therefore, by setting up the hypothesis as in (3), the consumer's risk (usually associated with the Type I error) is protected. Once the consumer's risk is restricted to, say, a level 5\% error, the agency such as the FDA leaves the pharmaceutical industry to determine the extent of manufacturer's risk via the type II error.

There are two additions to the work in the above context. First of all we have to have a prior structure for the parameters $\delta_{\mathrm{ij}}, \mu_{1}$ and $\sigma_{1}{ }^{2}$. Next we have to put our model in a bivariate context to account for the possible correlated response of the AUC and the Cmax. This will in turn give rise to an additional prior structure for some of the additional parameters.

We assume:

$$
\begin{aligned}
& \delta_{\mathrm{ij}} \sim \mathrm{N}\left(\mu, \sigma^{2}\right) \\
& \mu_{1} \sim \mathrm{N}\left(0, \sigma_{\mathrm{u}}^{2}\right) \\
& \sigma_{\mathrm{l}}^{2} \sim \mathrm{IG}(\mathrm{a}, \mathrm{b}),
\end{aligned}
$$

where IG refers to the inverted gamma 1 distribution. The hyperparameters $\left(\sigma_{\mathrm{u}}^{2}, \mathrm{a}, \mathrm{b}\right)$ are assumed to be known. We now put the $\mathrm{y}_{\mathrm{ijk}}$ in a bivariate normal context for the two responses of AUC and Cmax with a mean vector for the responses as well as a variance covariance structure. We then use Markov Chain Monte Carlo procedures to solve for the posterior parameters. If we let 1 denote the first column vector of AUC response and 2 denote the second column vector of Cmax responses, then in order to account for the sequence, $\mathrm{i}$, period, $\mathrm{k}$, and treatment effect for each subject, $\mathrm{j},\left(\mathrm{i}=1,2 ; \mathrm{j}=1,2, \cdots, \mathrm{n}_{\mathrm{i}} ; \mathrm{k}=1,2\right)$, we have the model in simplified terms for presentation:

$$
\mathrm{Y}[\mathrm{j}, 1: 2] \sim \mathrm{MVN}(\mathrm{u}[\mathrm{j}, 1: 2], \operatorname{Cov}[1: 2,1: 2])
$$

where MVN is multivariate normal, $\mathrm{u}$ denotes the mean vector and Cov denotes the variance covariance structure. Thus assuming a linear relationship between treatment response (AUC and Cmax) and the sequence, S, period, P, and treatment effect which we denote as alpha, we have for the jth subject, the AUC model,

$$
\mathrm{u}[\mathrm{j}, 1]=\text { beta }_{\mathrm{SEQ}} * \mathrm{seq}[\mathrm{j}, 1]+\text { beta }_{\text {PERIOD }} * \text { period }[\mathrm{j}, 2]+\mathrm{alpha} 1 * \operatorname{treat}[\mathrm{j}, 3]+\mathrm{s}[\mathrm{g}[\mathrm{j}], 1]
$$

where $s[\mathrm{~g}[\mathrm{j}]$,$] denotes the random subject effect. Likewise for the Cmax response vector we have$

$$
\mathrm{u}[\mathrm{j}, 2]=\text { beta }_{\text {SEQ }} * \text { seq }[\mathrm{j}, 1]+\text { beta } 2_{\text {PERIOD }} * \text { period }[\mathrm{j}, 2]+\text { alpha } 2 * \operatorname{treat}[\mathrm{j}, 3]+\mathrm{s}[\mathrm{g}[\mathrm{j}], 2] \text {. }
$$

This is from the notation provided by Ghosh and Gonen (2008).

The priors on the vectors in (5) are :

$$
\begin{aligned}
& \mathrm{u}[\mathrm{j}, 1: 2] \sim \operatorname{Normal}(0,1 / \mathrm{tau}) . \quad \operatorname{tau}=1000, \\
& \operatorname{Cov} 1[1: 2,1: 2]) \sim \text { Wishart }(\operatorname{var}=1, \operatorname{Cov}=0) .
\end{aligned}
$$

Although the entire program is not presented here, the correlation structure, $\rho$, for AUC and Cmax is coded as prior beta (including uniform) and will be presented in the "Results" section. The goal is to ultimately determine the posterior means for the $\mathrm{ABE}$ of $\mathrm{AUC}$ and Cmax, the sequence, period and treatment effects as well as the posterior mean of the correlation between the two response parameters AUC and Cmax.,

We consider a bioequivalence example from Balthasar (1999). The data (AUC and Cmax) are presented from a simulated cyclosporine bioequivalence study with 12 subjects. Apparently their data were simulated using a one compartment model. The author assumed the following values for pharmacokinetic parameters (mean \pm inter patient SD): bioavailability $0.3 \pm 0.4$, absorption rate constant $0.28 \mathrm{~h}-1 \pm 0.13$, clearance $53.8 \mathrm{~L} / \mathrm{h} \pm 21.4$, volume of distribution $301 \mathrm{~L} \pm 217$. Intra patient variances in model parameters were assumed as follows: bioavailability $(0.2 \mathrm{x}$ 
Al Bartolucci et al., Bayesian modeling of pharmaceutical data addressing the average effect of bivariate...

mean value $)^{2}$, absorption rate constant $(0.23 \mathrm{x} \text { mean value })^{2}$, volume of distribution and clearance $(0.1 \times$ mean values $)^{2}$. Data were simulated for a two-treatment, crossover study of $300 \mathrm{mg}$ of cyclosporine administered orally. The author performed the simulation assuming the same parameter values and variances for both study periods; consequently, there was no 'true' difference between the formulations in terms of their rate and extent of absorption. However, the simulated results lead to parameter values with moderately high values of intra patient variability in AUC (percent coefficient of variation: 19.3 percent) and in Cmax (percent coefficient of variation: 23 percent). These intra patient variabilities did agree well (Balthasar, 1999) with the experimental results of Kovarik et al.(1994) in their investigation of intra patient variability in cyclosporine pharmacokinetics .

\section{RESULTS}

The analysis of the data was done in two stages. We first examined the results in Table 1 under the assumption of independence of the AUC and the Cmax. Vague normal priors were assumed for the mean values of the responses, AUC and the Cmax, as well as the treatment and period effect. A posterior probability was generated for the average bioequivalence of the two responses. One can see that the posterior mean for the AUC is 7.215 and immediately below that result is the treatment and period effect on the bioequivalent response. Both the posterior credible regions for these parameters contain 0 indicating no effect on the overall result. The value of $\mathrm{P}(\mathrm{ABE})$ indicates that the posterior probability of average bioequivalence is about 0.638 which suggests pretty strong evidence for ABE. The results for Cmax are in the lower half of the table. One can see no strong treatment or period effect on the $\mathrm{ABE}$ for either the treatment or period in the design, the probability of $\mathrm{ABE}$ is around 0.558. In both cases of the $\mathrm{P}(\mathrm{ABE})$ the posterior credible regions default to $(0.0,1.0)$ using the Markov Chain Monte Carlo simulation results. One can see from Figure 1 there are clearly no effects of treatment on the ABE results for the AUC(Top) or the Cmax (Bottom). The "10000" noted in each plot indicates a 10,000 burn simulation for the MCMC results. The solution was actually attained with a more rapid convergence.. However we used the 10,000 as a standard. Thus the zero correlation results clearly supported the conclusion of bioequivalence. However, , the correlation between the two parameters of interest was quite high at about 0.869 , which indicates that perhaps one should consider a bivariate relationship between the Cmax and AUC and determine if the evidence of $\mathrm{ABE}$ is still substantiated. We now invoke equations (5) to (8) above applying our multivariate approach to this issue. Tables 2 and 3 give the results for both a prior uniform $(0,1)$ and prior Beta $(1,2)$ distribution on the prior correlation structure for the AUC and the Cmax. One can see from Table 2 for uniform $(0,1)$

Table 1 Posterior Results for Correlation $=0$

\begin{tabular}{|c|c|c|c|c|}
\hline Parameter & Mean & SD & Lower & Upper \\
& & & $2.5 \%$ & $97.5 \%$ \\
\hline AUC & 7.215 & 0.277 & 6.672 & 7.758 \\
\hline Treatment & -0.022 & 0.243 & -0.517 & 0.468 \\
\hline Period & -0.043 & 0.241 & -0.529 & 0.435 \\
\hline P(ABE) & 0.638 & 0.4805 & 0.0 & 1.0 \\
\hline Cmax & 4.72 & 0.256 & 4.22 & 5.231 \\
\hline Treatment & 0.072 & 0.263 & -0.461 & 0.539 \\
\hline Period & 0.066 & 0.260 & -0.588 & 0.453 \\
\hline P(ABE) & 0.558 & 0.497 & 0.0 & 1.0 \\
\hline
\end{tabular}
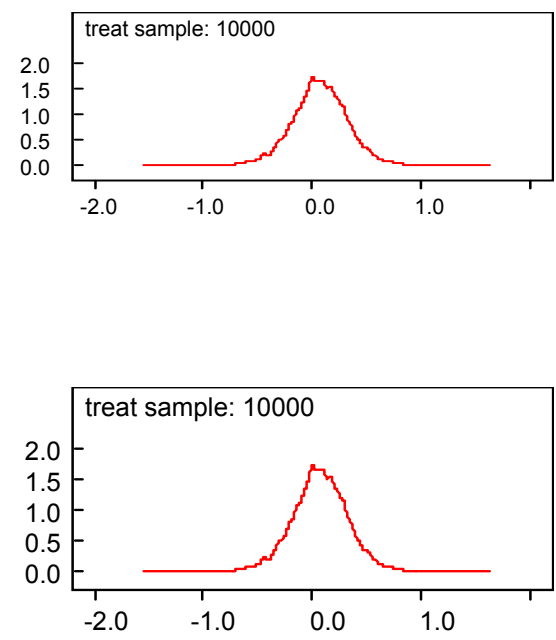

Figure 1. Posterior Densities of treatment effect on the ABE (AUC, Top) and ABE (Cmax, Bottom). 
Al Bartolucci et al., Bayesian modeling of pharmaceutical data addressing the average effect of bivariate...

that we have rows for the sequence effect as well as the posterior correlation, $\rho$, and the joint probability of bioequivalence $\mathrm{P}(\mathrm{ABT})$. One can also see that the posterior correlation here is about 0.572 and the joint $\mathrm{P}(\mathrm{ABT})$ is about 0.212 . The joint $\mathrm{P}(\mathrm{ABT})$ from Table 1 under the assumption of independence of the AUC and Cmax is about 0.356. Also unlike Table 1 there seems to be a period effect for the AUC in Table 2. Also the posterior mean (sd)

Table 2 Bivariate Posterior Results for prior uniform $(0,1)$ distribution the Correlation.

\begin{tabular}{|c|c|c|c|c|}
\hline Parameter & Mean & SD & Lower & Upper \\
& & & $2.5 \%$ & $97.5 \%$ \\
\hline AUC & 6.756 & 0.3961 & 5.968 & 7.546 \\
\hline Treatment & -0.047 & 0.219 & -0.478 & 0.409 \\
\hline Period & 1.136 & 0.464 & 0.225 & 2.077 \\
\hline Sequence & 0.899 & 0.555 & -0.145 & 2.035 \\
\hline P(ABE) & 0.405 & 0.491 & 0.0 & 1.0 \\
\hline Cmax & 4.135 & 0.336 & 3.468 & 4.798 \\
\hline Treatment & -0.071 & 0.182 & -0.432 & 0.295 \\
\hline Period & 0.077 & 0.186 & -0.282 & 0.453 \\
\hline Sequence & -0.018 & 0.215 & -0.437 & 0.422 \\
\hline P(ABE) & 0.444 & 0.497 & 0.0 & 1.0 \\
\hline P(ABT) & 0.212 & 0.409 & 0.0 & 1.0 \\
\hline$\rho$ & 0.572 & 0.299 & 0.032 & 0.986 \\
\hline & & & & \\
\hline & & & & \\
\hline & & & & \\
\hline
\end{tabular}

Table 3 Bivariate Posterior Results for prior $\operatorname{Beta}(1,2)$ distribution on the Correlation.

\begin{tabular}{|c|c|c|c|c|}
\hline Parameter & Mean & SD & Lower & Upper \\
& & & $2.5 \%$ & $97.5 \%$ \\
\hline AUC & 6.740 & 0.510 & 5.954 & 7.525 \\
\hline Treatment & -0.055 & 0.219 & -0.480 & 0.381 \\
\hline Period & 0.950 & 0.542 & -0.039 & 1.987 \\
\hline Sequence & 1.175 & 0.506 & 0.274 & 2.060 \\
\hline P(ABE) & 0.402 & 0.490 & 0.0 & 1.0 \\
\hline Cmax & 4.158 & 0.562 & 3.460 & 4.843 \\
\hline Treatment & -0.079 & 0.217 & -0.450 & 0.288 \\
\hline Period & 0.043 & 0.436 & -0.316 & 0.428 \\
\hline Sequence & -0.023 & 0.365 & -0.469 & 0.404 \\
\hline P(ABE) & 0.441 & 0.497 & 0.0 & 1.0 \\
\hline P(ABT) & 0.210 & 0.408 & 0.0 & 1.0 \\
\hline$\rho$ & 0.368 & 0.252 & 0.014 & 0.888 \\
\hline
\end{tabular}

difference from period 1 to period 2 in the AUC is $-0.09393(0.44)$ and the posterior difference for the Cmax is $0.1418(0.36)$.

One can see from Table 3 for the Beta $(1,2)$ prior on $\rho$ that we have a sequence effect but no period effect for AUC as in Table 2. Also the posterior correlation, $\rho$, is less than in Table 2. The joint $\mathrm{P}(\mathrm{ABT})$ is relatively unchanged from Table 2. Also the posterior mean (sd) difference from period 1 to period 2 in the AUC is -0.1108 (0.45) and the posterior difference for the Cmax is $-0.1599(0.43)$. As we attempted to increase the prior Beta scale and the shape parameters for the correlation structure our MCMC program became unstable in the sense that the results were non robust outside the ranges we attempted which probably is an indication that Tables 1,2 and 3 are constructed for a realistic range for the correlation hyper parameters. The overall result clearly shows that one must take into account the correlation of these two parameters, AUC and Cmax when reporting bioequivalent results. The $\mathrm{P}(\mathrm{ABE})$ and $\mathrm{P}(\mathrm{ABT})$ are certainly sensitive to this consideration. 
Al Bartolucci et al., Bayesian modeling of pharmaceutical data addressing the average effect of bivariate...

\section{DISCUSSION AND CONCLUSIONS}

There certainly has been sufficient discussion (Ghosh and Gonen, 2008) about the mathematical caution that one should take before attempting to present bioequivalent results under the assumption of the independence of the parameters of interest such as the AUC, Cmax, Tmax or others. The attempt here computationally was twofold. First of all we wanted to apply this concern to a data set of interest and secondly we wished to examine the behavior of the MCMC approach to the problem. In the case of assumed correlation of 0 we naturally were able to generate with relative ease the results of Table 1 giving the posterior estimates of the parameters of interest as well as the $\mathrm{P}(\mathrm{ABE})$ for each. The MCMC procedure was stable and yielded results that were in line with the original author's presentation (Balthasar,1999) from a non Bayesian perspective and under the assumption of independence of the AUC and Cmax. Next we utilized the MCMC approach of Ghosh and Rosner (2007) and Ghosh and Gonen (2008) keeping within the framework of the bivariate consideration of the parameters. That particular program was a challenge in that the input format was not clearly outlined in their work. Nonetheless the script as presented for the ensuing results discussed in our work here was very well done and once one gets beyond the input challenge for a crossover design the data as reported was certainly sensible. One can see that taking into account prior correlation does impact on the overall $\mathrm{P}(\mathrm{ABE})$ as well as the $\mathrm{P}(\mathrm{ABT})$. The results were consistent and robust with respect to the prior non informative input of most of the parameters in that the treatment effect was negligible across the periods of the experiment. However, one has to exercise caution when one inputs the prior structure for the correlation because the convergence to a solution requires much more burn iterations if one is not within a realistic range of the prior correlation. This was obvious when conducting this analysis as one can converge to a solution fairly quickly within no more that 5000 to 10,000 iterations for realistic priors on the correlation such as the uniform $(0,1)$ and beta scale 1 and shape no greater than 2 . Of course one should always conduct sensitivity analyses when doing this type of analysis.

\section{REFERENCES}

Balthasar, J.P.( 1999). Bioequivalence and bioequivalence testing. American Journ.of Pharm Education. 63, 194-198.

Bartolucci, AA. Bae, S.J. and Singh, K.P. (2008). Establishing a Bayesian predictive survival model adjusting for random effects. Mathematics and Computers in Simulation. 78, 328-334.

Berger, R.L. And Hsu, J.C. (1996). Bioequivalence trials, intersection union tests and equivalence confidence Sets(with discussion). Statistical Science. 11, 283-319.

Bolton, S. (1991). Outliers - examples and opinions. Presented at Bioavailability/Bioequivalence Pharmacokinetic and Statistical Considerations. Drug Information Association, Bethesda, MD.

Breslow, N. (1990). Biostatistics and Bayes. Statistical Science. 5, 269-298..

Chow, S.C. and Liu, J.P.(2000). Design and analysis of Bioavailability and Bioequivalence Studies. ( $2^{\text {nd }}$ ed.). Marcel Dekker. New York.

Food and Drug Administration. Bioavailabilitry and Bioequivalence Studies for Orally Administered Drug Products (2003) . FDA. CDER. http://www.fda.gov/cder/guidance

Ghosh, P. and Gonen, M. (2008). Bayesian Modeling of multivariate average bioequivalence. Statistics in Medicine.. 27, 2402-2419.

Ghosh, P. and Rosner,G.L. (2007). A semiparametric approach to average bioequivalence. Statistics in Medicine.. 26, 1224-1236.

Manly, B.F. (2004). One sided tests of bioequivalence with non normal tests and unequal variance. Journal of Agricultural Biological and Environmental Statistics. 9, 270-281.

Kovarik, J.M., Mueller, E.A., van Bree, J.B., Tetzloff, W. and Kutz, K.,(1994) Reduced inter- and intra individual variability in cyclosporine pharmacokinetics from a microemulsion formulation. J. Pharm. Sci., 83, 444-446.

Rogers, J. I., Howard, K. I. and Vessy, J. T. (1993). Using significance tests to evaluate equivalence between two experimental groups. Psychological Bulletin. 113, 553-556.

Roy, T. (1997). Calibrated nonparametric confidence sets. Journal of Mathematical Chemistry 21, 103-109

Westlake, W. J. (1981). Bioequivalence testing - a need to rethink [reader reaction response]. Biometrics 37, 591593. 